University of Nebraska - Lincoln

DigitalCommons@University of Nebraska - Lincoln

2009

\title{
The minority spin surface bands of $\mathrm{CoS}_{2}(001)$
}

Ning Wu

University of Nebraska-Lincoln

Renat F. Sabirianov

University of Nebraska at Omaha, rsabirianov@mail.unomaha.edu

Wai-Ning Mei

University of Nebraska at Omaha, physmei@unomaha.edu

Yaroslav B. Losovyj

Louisiana State University at Baton Rouge, ylozovyy@indiana.edu

N. Lozova

Louisiana State University

See next page for additional authors

Follow this and additional works at: https://digitalcommons.unl.edu/physicsdowben

Part of the Physics Commons

Wu, Ning; Sabirianov, Renat F.; Mei, Wai-Ning; Losovyj, Yaroslav B.; Lozova, N.; Manno, M.; Leighton, C.; and Dowben, Peter A., "The minority spin surface bands of $\mathrm{CoS}_{2}(001) "$ (2009). Peter Dowben Publications.

243.

https://digitalcommons.unl.edu/physicsdowben/243

This Article is brought to you for free and open access by the Research Papers in Physics and Astronomy at DigitalCommons@University of Nebraska - Lincoln. It has been accepted for inclusion in Peter Dowben Publications by an authorized administrator of DigitalCommons@University of Nebraska - Lincoln. 


\section{Authors}

Ning Wu, Renat F. Sabirianov, Wai-Ning Mei, Yaroslav B. Losovyj, N. Lozova, M. Manno, C. Leighton, and Peter A. Dowben 


\title{
The minority spin surface bands of $\mathrm{CoS}_{2}(001)$
}

\author{
Ning Wu ${ }^{1}$, R F Sabirianov ${ }^{2}$, W N Mei $^{2}$, Ya B Losovyj ${ }^{1,3}, \mathbf{N L o z o v a}^{3}$, \\ M Manno ${ }^{4}$, C Leighton ${ }^{4}$ and P A Dowben ${ }^{1}$ \\ ${ }^{1}$ Department of Physics and Astronomy and the Nebraska Center for Materials and \\ Nanoscience, University of Nebraska-Lincoln, Lincoln, NE 68588-0111, USA \\ ${ }^{2}$ Department of Physics, University of Nebraska at Omaha, Omaha, NE 68182-0266, USA \\ ${ }^{3}$ Center for Advanced Microstructures and Devices, Louisiana State University, \\ 6980 Jefferson Highway, Baton Rouge, LA 70806, USA \\ ${ }^{4}$ Department of Chemical Engineering and Materials Science, University of Minnesota, \\ Minneapolis, MN 55455, USA
}

Received 15 February 2009, in final form 21 May 2009

Published 3 July 2009

Online at stacks.iop.org/JPhysCM/21/295501

\begin{abstract}
Angle-resolved photoemission was used to study the surface electronic band structure of high quality single crystals of ferromagnetic $\mathrm{CoS}_{2}$ (below $120 \mathrm{~K}$ ). Strongly dispersing Co $\mathrm{t}_{2 \mathrm{~g}}$ bands are identified along the $\langle 100\rangle k_{\|}$direction, the $\bar{\Gamma}-\bar{X}$ line of the surface Brillouin zone, in agreement with model calculations. The calculated surface band structure includes corrections for the previously determined surface structure of $\mathrm{CoS}_{2}(001)$ and is in general agreement with the experimental photoemission spectra in the region of the Fermi level. There is evidence of the existence of several minority spin surface states, falling into a gap of the projected minority spin bulk $\mathrm{CoS}_{2}(001)$ band structure.
\end{abstract}

\section{Introduction}

The pyrite-type transition metal compound $\mathrm{CoS}_{2}$ is an itinerant electron ferromagnet and is predicted, in ground state band structure calculations [1-7], to be close to half-metallic. Although some minority spin states are present at the Fermi level in bulk band structure calculations (e.g. [7]), the system is predicted to be relatively highly spin-polarized. The measured saturation magnetizations $\left(0.74 \mu_{\mathrm{B}} / \mathrm{CoS}_{2}\right.$ [8]; $\left.0.85 \mu_{\mathrm{B}} / \mathrm{Co}[9] ; 0.87 \mu_{\mathrm{B}} / \mathrm{CoS}_{2}[10,11]\right)$ lie close to, but less than, the $1.0 \mu_{\mathrm{B}} / \mathrm{Co}$ expected of ideal half-metallic $\mathrm{CoS}_{2}$, consistent with these calculations. The measured transport spin polarizations of $56-64 \%$, as determined from pointcontact Andréev reflection for $\mathrm{CoS}_{2}$ [10-12], are actually quite similar to the measured polarization (40-50\%) of nominally half-metallic NiMnSb [13-18]. Much higher electron spin polarization values have been measured for $\mathrm{NiMnSb}(100)$ using spin-polarized inverse photoemission $[19,20]$ but in regions of the surface Brillouin zone away from any surface state Fermi level crossing for $\mathrm{NiMnSb}(100)$. As is common for spin-polarized inverse photoemission, the measurements for $\mathrm{NiMnSb}(100)$ were taken at normal electron incidence or along the $\bar{\Gamma}$ point (the surface Brillouin zone center) corresponding to a surface parallel wavevector equal to zero $[19,20]$ and the values of the polarization cannot be easily compared to the polarization determined by Andréev reflection [21-23]. Indeed, one of the serious problems associated with the study of high polarization materials is that the value of the spin polarization depends upon the measurement, not simply the material [22, 23].

Considering the proximity of the bulk minority spin state to the Fermi level and the overall polarization, $\mathrm{CoS}_{2}$ actually resembles the much touted $\mathrm{La}_{0.65} \mathrm{Sr}_{0.35} \mathrm{MnO}_{3}[24,25]$, although $\mathrm{CoS}_{2}$ is structurally and electronically simpler. Significantly, $\mathrm{CoS}_{2}$ can also be alloyed with the narrow band gap semiconductor $\mathrm{FeS}_{2}$ to 'fine-tune' the Fermi level position, thus generating the highly spin-polarized ferromagnet $\mathrm{Fe}_{1-x} \mathrm{Co}_{x} \mathrm{~S}_{2}$, which more closely resembles an ideal ground state half-metallic ferromagnet [4-7, 11].

Device utilization of highly spin-polarized ferromagnets (e.g. for spin injection) requires fabrication of a heterostructured device architecture. Interface and surface effects then become critical. Minority spin surface states in the nominally half-metallic systems are well known [21, 26-37] and can 'evolve' into minority spin interface states in the presence of overlayers [21, 32-37]. The existence of such spin-polarized 
surface-localized electronic states may have profound consequences; the introduction of interfaces can increase the minority spin population at the Fermi level, with the consequent loss of half-metallic character, even near $T=0$.

Although not completely half-metallic (as discussed above), the bulk band structure of $\mathrm{CoS}_{2}$ projected on to the (001) surface retains a large band gap in the vicinity of the Fermi level for one spin channel (in the occupied minority spin density of states just below $E_{\mathrm{F}}$ ) [7]. Although the Curie temperature of pyrite structure $\mathrm{CoS}_{2}$ is rather low $\left(T_{\mathrm{C}} \sim\right.$ $120 \mathrm{~K}$ [10]), thus placing limits on the device applications of this material, the investigation of the surface band structure of $\mathrm{CoS}_{2}$-based ferromagnets (i.e. pure $\mathrm{CoS}_{2}$ or the $\mathrm{Co}_{1-x} \mathrm{Fe}_{x} \mathrm{~S}_{2}$ system) will allow us to develop a better understanding of the undesirable minority spin surface states in highly spinpolarized materials. Surface band structure studies are clearly required to fully assess the role of the broken symmetry in the $z$ direction due to the surface, as well as the role of the surface relaxation(s) in shifting the position of the minority spin bands relative to the Fermi level. This is the goal of the current study, which has utilized $\mathrm{CoS}_{2}(001)$ single crystals. The stoichiometric $\mathrm{CoS}_{2}(001)$ single crystal surfaces can be and have been prepared by cleavage and these are surfaces where we have characterized the details of the surface lattice relaxation and surface structure in prior work. Effects such as surface symmetry breaking were found to be important in $\mathrm{Fe}_{3} \mathrm{O}_{4}$, for example, and may result in shifts of the minority spin channel towards, and even across, the Fermi level [31].

\section{Experimental details}

The $\mathrm{CoS}_{2}(001)$ single crystals (several millimeters in diameter) were cleaved, as detailed in a previous publication [7, 38], which provided low energy electron diffraction (LEED) patterns characteristic of a highly ordered surface [38], as indicated in figure 1. Surface composition and order are seen to be strongly dependent upon surface preparation [37], but the samples appear to be single crystals with no evidence of twinning or grain boundaries in the LEED or x-ray diffraction. The data reported here are for the stoichiometric surface, with the surface termination in the dense Co-S plane [36], illustrated schematically in figure 1 .

We present here spin-integrated experimental band mapping measurements, primarily because single crystalline thin films of $\mathrm{CoS}_{2}(001)$, with known surface stoichiometry, are not presently available. Spin-polarized photoemission and inverse photoemission band mapping measurements typically require ultra-thin film surfaces of known surface stoichiometry. Successful spin-polarized photoemission and inverse photoemission measurements require high values of remanent magnetization along with small saturation magnetization fields: the latter properties are characteristic of very thin single crystal films and are rare in bulk single crystals. As bulk single crystals, the rapid magnetic saturation of the samples in opposite directions which is necessary for spin-polarized photoemission or spin-polarized inverse photoemission studies has been difficult to successfully and reproducibly apply to our $\mathrm{CoS}_{2}(001)$ samples thus far. We have opted for higher resolution spin-integrated
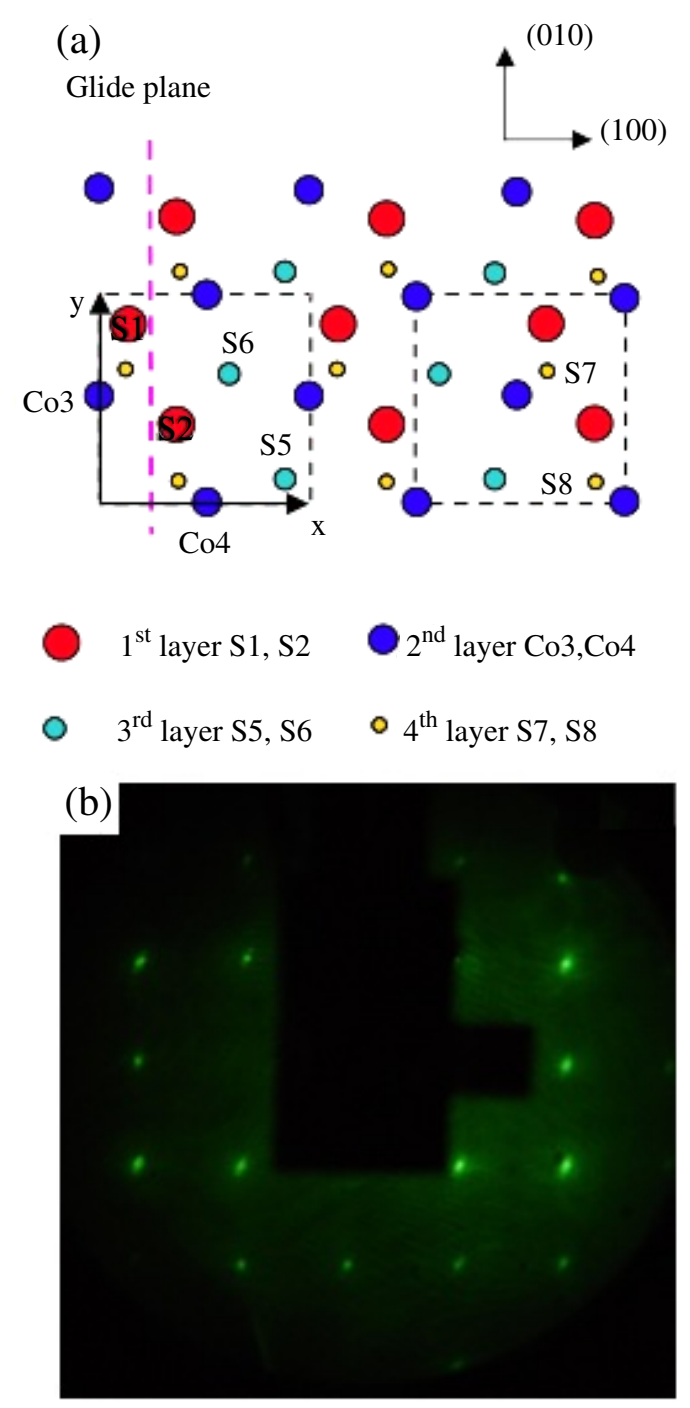

Figure 1. A schematic diagram of the $\mathrm{CoS}_{2}(100)$ surface $1 \mathrm{~S}$-termination as viewed from the top (a), adapted from [38], and the LEED image of the $\mathrm{CoS}_{2}(100)$ surface, at an electron kinetic energy of $111 \mathrm{eV}$ (b).

(This figure is in colour only in the electronic version)

photoemission and comparison with theory of surfaces for which the surface structure and composition has been well established $[38,39]$. Nevertheless, this represents one of very few surface band structure determinations of a nominally halfmetallic ferromagnet or very high spin polarization material.

The majority of the angle-resolved photoemission spectra were obtained using plane-polarized synchrotron light dispersed by a $3 \mathrm{~m}$ toroidal grating monochromator (TGM) [40] at the Center for Microstructures and Devices (CAMD). These measurements were made in a UHV chamber employing a hemispherical electron analyzer with an angular acceptance of $\pm 1^{\circ}$, as described elsewhere [40]. The combined resolution of the electron energy analyzer and monochromator was about $80 \mathrm{meV}$ [40]. The photoemission experiments were undertaken with a light incidence angle of $45^{\circ}$ with respect to the surface normal. All binding energies are referenced to the Fermi level, as determined from clean gold. The surface 
band mapping was undertaken at about $90 \mathrm{~K}$, below the Curie temperature of about $120 \mathrm{~K}$ [10].

\section{The surface band structure}

In the overall density of states of the $\mathrm{CoS}_{2}(001)$ surface, the states with strong Co d-band weight reside near the Fermi level [1-7]. The wider $\mathrm{S} 3 \mathrm{p}$ bands at -4 and $-6 \mathrm{eV}$ binding energies (defined here as $E-E_{\mathrm{F}}$ ) are described as $\mathrm{Co}-\mathrm{S}$ hybridized bands as has been demonstrated by the resonance photoemission at the super Coster-Kronig Co $3 p \rightarrow$ $3 \mathrm{~d}$ transition [7]. $\mathrm{CoS}_{2}$ is largely an itinerant $3 \mathrm{~d}$ electron ferromagnet, and the occupied states within $2 \mathrm{eV}$ binding energy of the Fermi energy exhibit very little dependence on photon energy [7]. This absence of photon energy dependence suggests conservation of two dimensionality of state, indicating that these features, as observed in photoemission at lower photon energies [7], are most likely dominated by surface states (surface-localized states that are at binding energies that fall into a gap of the projected bulk band structure) or surface resonances (surface-localized states that are at binding energies that are shared by bands of the bulk band structure). The higher resolution photoemission spectra focus on the Co 3d bands, not the hybridized bulk S 3p weighted bands studied in prior investigations [7].

Figure 2 illustrates the dependence of photoemission spectra of Co $3 \mathrm{~d}$ bands on emission angle along the $\langle 100\rangle$ direction of the surface, with electrons collected at different angles from $-10^{\circ}$ to $+16^{\circ}$, at a photon energy of $43 \mathrm{eV}$. Due to the crystal field, the $3 \mathrm{~d}$ band of the octahedrally coordinated Co splits into two subbands: the $\mathrm{t}_{2 \mathrm{~g}}$ and $\mathrm{e}_{\mathrm{g}}$ bands of the bulk band structure. The sharp photoemission peak around $-0.8 \mathrm{eV}\left(E-E_{\mathrm{F}}\right)$ is attributed to, at least two strong surface weighted states overlapping with, or placed very close to the fully occupied bulk Co $3 \mathrm{~d}$ weighted $\left(\mathrm{t}_{2 \mathrm{~g}}\right)$ minority spin bands. The partially occupied Co $3 \mathrm{~d}$ (minority spin bulk $\mathrm{e}_{\mathrm{g}}$ ) band can be seen to contribute to the shoulder of the prominent Co peak near the Fermi level at a binding energy of -0.2 to $-0.3 \mathrm{eV}\left(E-E_{\mathrm{F}}\right)$, and can been seen as a separate feature in the inset photoemission spectra taken at slightly higher photon energy (46 eV versus $43 \mathrm{eV}$ ) with improved statistics. This photoemission feature at -0.2 to $-0.3 \mathrm{eV}\left(E-E_{\mathrm{F}}\right)$ contains strong surface weight, as demonstrated by the conservation of the two dimensionality of state and indicated by the absence of photon energy dependence in this region of the spectra. Such an assignment of surface weight to the features closest to the Fermi level is also supported by the fact that the features in this region of the spectra (near the Fermi level) are also in fact very sensitive to contamination.

In fact, for higher resolution photoemission taken with a slightly higher photon energy $(46 \mathrm{eV})$, analysis of the spectra indicate that there are at least five components in the region where there is strong Co $3 \mathrm{~d}$ weight, as indicated in the inset to figure 2. As discussed later, these strong Co $3 \mathrm{~d}$ weight components of the photoemission spectra in the region near the Fermi level are consistent with the surface band structure calculations.

The photoemission data of Takahashi and co-workers [41] also showed that the Co $3 \mathrm{~d}$ band can be resolved into

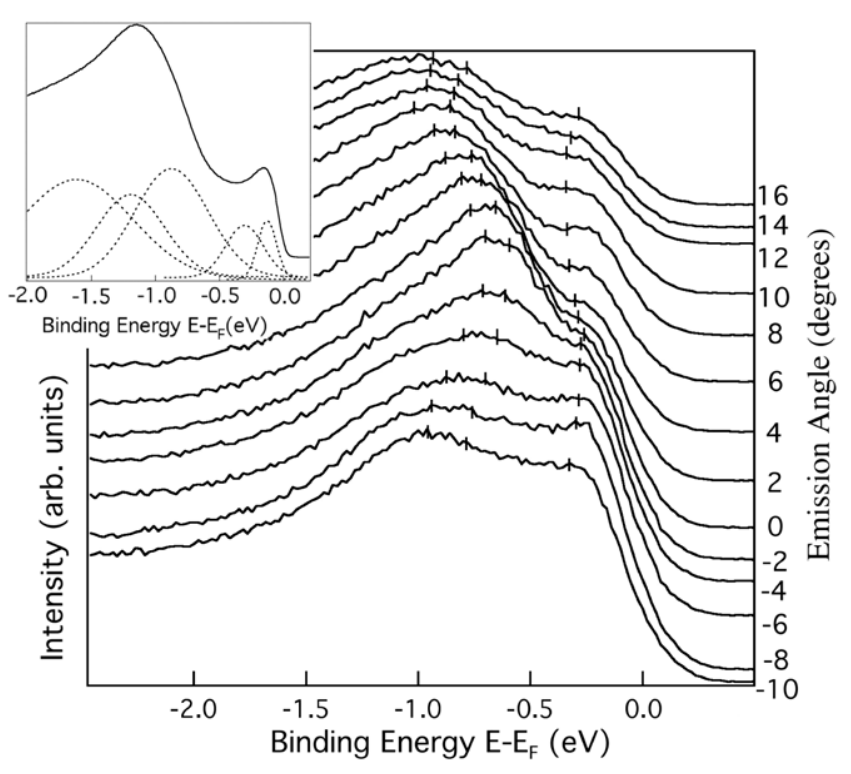

Figure 2. The photoemission spectra of $\mathrm{CoS}_{2}(001)$ for different emission angles from $-10^{\circ}$ to $+16^{\circ}$, taken at a photon energy of $43 \mathrm{eV}$. The tick marks indicate the contributions to the spectra with strong surface weight i.e. conservation of two dimensionality of state (features that show little photon energy dependence). The inset shows a photoemission spectrum taken at $46 \mathrm{eV}$ photon energy and with a larger accumulation of counts collected along the surface normal. For the inset some of the components of the photoemission spectra are indicated.

two subbands, in the vicinity of $-1 \mathrm{eV}$ binding energy $\left(E-E_{\mathrm{F}}\right)$ and the Fermi level. The bulk $3 \mathrm{~d} \mathrm{e}_{\mathrm{g}}$ bands are located around $-0.3 \mathrm{eV}$ binding energy and, therefore, the experimental splitting between the bulk $3 \mathrm{~d}_{\mathrm{g}}$ bands and $3 \mathrm{~d} \mathrm{t}_{2 \mathrm{~g}}$ bands amounts to $0.5 \mathrm{eV}$. Although generally consistent with theory [1, 2] and previous work [41], these band placements are compromised by the strong surface weight of the bands observed here in photoemission.

The Co $3 \mathrm{~d}$ weighted bands do exhibit dispersion with changing surface wavevector, as seen in figure 2 . The parallel component of the crystal wavevector $k_{\|}$can be derived as follows from the kinetic energy and the emission angle:

$$
k_{\|}=\left(\frac{2 m}{\hbar^{2}} E_{\mathrm{kin}}\right)^{1 / 2} \sin (\theta) .
$$

From the dispersion of the bands with wavevector parallel with the surface, $k_{\|}$, evident in the photoemission spectra as a function of emission angle (figure 2), we have mapped the spin-integrated band structure along the $\bar{\Gamma}-\overline{\mathrm{X}}$ direction of the surface Brillouin zone. The experimental photoemission remains complicated by the fact that several Co $3 \mathrm{~d}$ weighted subbands exist that are not cleanly resolved in our photoemission spectra of the $\mathrm{CoS}_{2}(001)$ surface, as discussed below.

The experimental band structure is shown in figure 3 along the $\langle 100\rangle$ direction, or from $\bar{\Gamma}$ to $\bar{X}$ of the surface Brillouin zone. The normal emission angle corresponds to the $\bar{\Gamma}$ point in the surface Brillouin zone. Based on the surface lattice parameter of $5.51 \AA$ [36], the edge of the Brillouin zone 


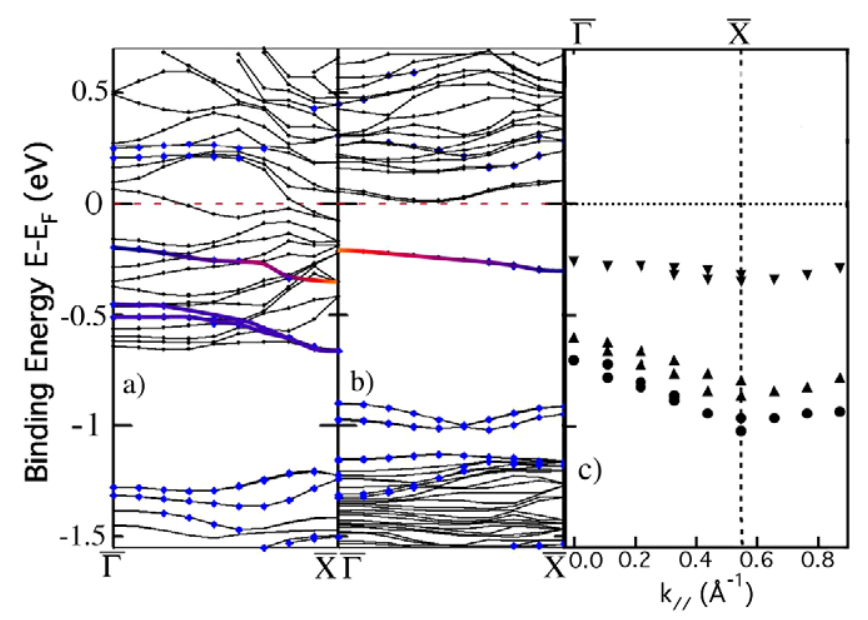

Figure 3. Band structure of the $\mathrm{Co}_{18} \mathrm{~S}_{36}$ slab composed of 18 composite layers of $\mathrm{CoS}_{2}$, along the $\bar{\Gamma}$ to $\overline{\mathrm{X}}$ direction with the calculated majority spin (panel (a)) and minority spin (panel (b)). Bands strongly localized at the surface, that is bands with more than $40 \%$ weight residing in the top one-Co and two-S layer, are indicated by the blue dots. Bands where the weight has decreased at the surface (i.e. dropped below $40 \%$ ) but retains strong weight in the near subsurface to $20 \%$ or more are expressed in red. We find that band weight can shift from surface to subsurface as a function of wavevector as indicated (see text). A portion of the experimental surface band structure of $\operatorname{CoS}_{2}(001)$ along the $\langle 100\rangle$ direction, derived from photoemission spectra taken at a photon energy of $43 \mathrm{eV}$, is shown in panel (c), with the close lying bands distinguished by the $(\boldsymbol{\Delta})$ symbols.

$\left(k_{\|}=0.57 \AA^{-1}\right)$ falls at an emission angle of about $10.6^{\circ}$ for a photon energy of $43 \mathrm{eV}$ and is thus consistent with the angleresolved photoemission which shows the Brillouin zone edge at a similar emission angle (figure 2) and wavevector (figure 3 ). The photoemission feature with a binding energy of -0.6 to $-0.7 \mathrm{eV}\left(E-E_{\mathrm{F}}\right)$ at the $\bar{\Gamma}$ point (the surface Brillouin zone center) disperses to greater binding energies of around -0.85 to $-0.95 \mathrm{eV}\left(E-E_{\mathrm{F}}\right)$ at the $\overline{\mathrm{X}}$ point (the surface Brillouin zone edge), while the width of this feature clearly increases as well. The variations in the width of this photoemission feature may in part be a consequence of multiple unresolved majority and minority spin components, as indicated in the possible scenario for the curve fitting analysis provided in the inset to figure 2 .

The photoemission feature placed even closer to the Fermi level also disperses away from the Fermi level with increasing wavevector along the $\bar{\Gamma}-\overline{\mathrm{X}}$ high symmetry line of the surface Brillouin zone. In addition, high resolution photoemission taken using a Scienta electron energy analyzer [41] $(\Delta E=$ $15 \mathrm{meV}$ ) in the region of the Fermi level suggests a decrease in the density of states near the Fermi level, consistent with the surface Brillouin zone, as indicated in figure 4. These high resolution results indicating a change in the density of states at the Fermi level with increasing wavevector, taken at a photon energy of $21.2 \mathrm{eV}$ (helium I), have been repeated at other photon energies $(36 \mathrm{eV})$ but because of a reduction of resolution at the high photon energies they are qualitatively similar but not as dramatic as those shown in figure 4 .

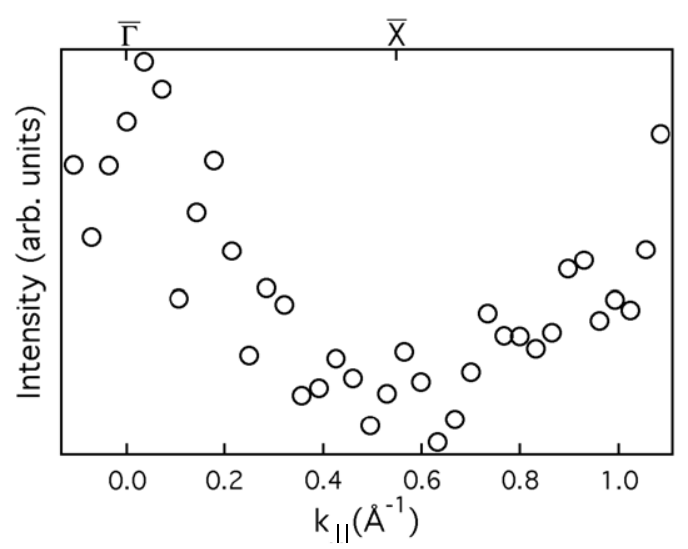

Figure 4. The intensity change taken with high resolution photoemission [42] $(\Delta E=15 \mathrm{meV}$ and a photon energy of $21.2 \mathrm{eV})$ in the vicinity of the Fermi level, indicating the possibility of a Fermi level crossing near $k_{\|}=0$ or $\bar{\Gamma}$.

\section{The calculated surface band structure}

To correctly model minority spin surface-localized electronic states, i.e. surface states and resonances, it is essential that spin-polarized electronic structure calculations fully take into account the details of the possible surface reconstructions and surface segregation. First-principle calculations of a $\mathrm{Co}_{18} \mathrm{~S}_{36}$ slab (18 composite layers implies $18 \mathrm{Co}$ and $36 \mathrm{~S}$ ) performed using the projected augmented plane wave method for a stable surface of $\mathrm{CoS}_{2}$ termination show good overall agreement with dynamical scattering analysis of the experimental LEED I(V) data [38]. The calculated band structure for the (001) surface includes the corrections for the known surface relaxation; the surface $\mathrm{S}$ atoms move outwards towards the vacuum, while the subsurface Co atoms move towards the bulk, by approximately 0.03 and $0.11 \AA$, respectively. The $\mathrm{S}$ atoms in the third sublayer relax outwards by about $0.12 \AA$ (details of calculations and discussion on the structural relaxation can be found in [38, 39]) thus providing an indication of a strong S-S dimer bond and a dense surface region. Due to the presence of the surface, the symmetry of the slab is reduced from the $f c c$ bulk periodic lattice symmetry.

The calculated $\mathrm{CoS}_{2}$ band structure along the $\bar{\Gamma}-\overline{\mathrm{X}}$ line of the surface Brillouin zone of the (001) surface is shown in figure 3 (panels (a) and (b)). Those bands with strong surface weight have been highlighted. In figure 3, those bands found to be strongly localized at the surface region, that is to say that more than $40 \%$ of their spectral weight resides in the top-surface layer composed of the one-Co and two-S subplanes, are indicated by blue dots in figures 3(a) and (b). Bands with continued strong surface weight, where the spectral contribution to the surface layer has dropped below $40 \%$ yet still remains around $30 \%$ are indicated by the red lines in figures 3(a) and (b). In the calculated $\mathrm{CoS}_{2}(001)$ surface band structure, those regions indicated by the red lines typically are where more of the band weight has shifted to the immediate subsurface Co plane. In bands with strong immediate subsurface layer weight (indicated by the red), the spectral density associated with the immediate subsurface Co 
plane may have probabilities that are as low as $5 \%-10 \%$, but in fact the overall subsurface spectral weight can increase to around $20 \%$ when the two-S subplanes are considered, and residual weight in the surface layers persists as well in these regions of the surface Brillouin zone.

With regard to surface weight, there are differences between the (001) band structure for majority and minority spins. The majority band, with a binding energy of around $-0.3 \mathrm{eV}\left(E-E_{\mathrm{F}}\right)$, illustrated in figure 3(a), starts from the $\bar{\Gamma}$ point with about $44 \%$ of the spectral weight density residing on the top layer. With increasing wavevector, this majority spin band has a surface spectral weight that drops below $40 \%$ in the middle of the surface Brillouin zone between $\bar{\Gamma}-\bar{X}$ and decreases in surface weight to about $34 \%$ at the surface Brillouin zone edge or $\overline{\mathrm{X}}$ point. The band is shown with more blue at the Brillouin zone center and the color is shown in figure 3(a) with increasing red highlighting at the Brillouin zone edge to indicate the change in the surface weight of the band from the surface towards the immediate subsurface layers. The corresponding minority band (panel (b)) also has a similar wave-vector-dependent band dispersion between $\bar{\Gamma}-\bar{X}$, starting again with a binding energy of around $-0.3 \mathrm{eV}\left(E-E_{\mathrm{F}}\right)$ from the $\bar{\Gamma}$ point. Unlike the majority spin component, the occupied minority spin band near the Fermi level has surface weight that increases with increasing wavevector. This minority spin band, with a binding energy of around $-0.3 \mathrm{eV}$, starts with a probability of about $33 \%$ that can be assigned to the surface layer, but with a strong subsurface layer contribution, as indicated in figure 3(b) in red. With increasing wavevector, the surface layer weight of this band increases to rise above $40 \%$ in the middle of the surface Brillouin zone (between $\bar{\Gamma}-\bar{X}$ ), and finishes at the Brillouin zone edge ( $\overline{\mathrm{X}}$ point), with about $43 \%$ of the band weight at the surface. Thus this minority spin band placed at around $-0.3 \mathrm{eV}$ $\left(E-E_{\mathrm{F}}\right)$ is shown in blue near the Brillouin zone edge.

The comparison with the experimental $k_{\|}$dispersions from $\bar{\Gamma}$ to $\overline{\mathrm{X}}$ indicates that the photoemission spectra are indeed dominated by surface weighted bands, although there is a very small shift of about $0.1 \mathrm{eV}$ to greater binding energies in the experiment, as is often the case in such comparisons with theory. The surface state and surface resonance band dispersions in theoretical and experimental band mappings are otherwise in general agreement. The model spin-polarized band structure calculations show that a well isolated surface state band appears near the Fermi level at binding energies of $-0.3 \mathrm{eV}$ (in agreement with the band observed at -0.2 to $-0.3 \mathrm{eV}$ in experiment) in the band gap of the projected minority states at the $\bar{X}$ point. This is a true surface state in the minority spin band structure. We observe a similar surface weighted band within the majority density of states, but this does not fall into a gap of the projected bulk band structure, and is thus a surface resonance. Because the majority spin surface resonance and minority spin surface state, at -0.2 to $-0.3 \mathrm{eV}$ binding energy, have such similar weights, binding energies, and dispersion, it appears as though this smaller binding energy surface band has no intra-atomic exchange splitting. Careful analysis of the experimental photoemission spectra taken at the surface Brillouin zone center (as in the inset the figure 2) suggests the presence of other majority spin bands with strong surface weight as well, i.e. majority spin bands that are evident in the model calculations. These other surface weight bands that contribute to the photoemission spectra at about $-0.5 \mathrm{eV}$ binding energy $\left(E-E_{\mathrm{F}}\right)$ do have overlap with the bulk bands.

Several more surface weighted bands are observed at binding energies of $-0.9 \mathrm{eV}$ below the Fermi level in the minority spin band structure and at -1.2 to -1.5 ( $E-$ $\left.E_{\mathrm{F}}\right)$ in both the minority and majority spin band structure. These calculated surface weighted bands generally follow the dispersion and binding energy with wavevector seen in the photoemission experiments. Direct comparison with experiment is difficult as there are complications as a result of the contributions from the bulk band structure to the experimental photoemission spectra. Overall, the increase in binding energy with increasing wavevector is similar in both experiment and theory, with the greatest binding energies near the $\bar{X}$-point or surface Brillouin zone edge. There are differences between experiment and theory at the surface Brillouin zone edge, but these bands are not true surface states and do not fall into a gap of the projected bulk band structure.

The minority spin surface band companions to the majority spin surface weighted bands at about $-0.5 \mathrm{eV}$ binding energy $\left(E-E_{\mathrm{F}}\right)$ tend to dominate the photoemission spectra more at the surface Brillouin zone edge and exhibit somewhat higher binding energies $(-0.8$ to $-1.0 \mathrm{eV})$. Here the binding energies of the majority $(-0.5 \mathrm{eV}$ binding energy) and minority spin $(-0.8$ to $-1.0 \mathrm{eV})$ components differ as a result of the ground state exchange splitting of this surface weighted band, but appear to be very difficult to resolve in experiment near the center of the Brillouin zone. This minority spin band at about -0.8 to $-1.0 \mathrm{eV}\left(E-E_{\mathrm{F}}\right)$ certainly contributes to the observed photoemission density of states near the Brillouin zone edge.

The surface weighted bands of majority spin along the $\bar{\Gamma}-\bar{X}$ direction with binding energies greater than $-1.2 \mathrm{eV}$ (figure 3(a)) cannot be separately distinguished from similar minority spin bands at similar binding energies $\left(E-E_{\mathrm{F}}\right)$ nor the overlapping bulk bands in the experimental spectra. The experimental photoemission is complicated by contributions from the bulk, the contributions from both majority spin and minority spin bands, and finite temperature effects, leading to a broad photoemission feature in the region of $-1.5 \mathrm{eV}$ binding energy (inset to figure 2). While it is unsurprising that there are differences between experiment and theory, there are regions where photoemission and the calculated surface band structure agree, particularly at the surface Brillouin zone edge.

\section{Conclusions}

In summary, model surface band structure calculations indicate that there exists a true minority spin surface state at a binding energy below the Fermi energy, and that the predicted surface weighted Co $3 \mathrm{~d}$ bands appear to match experiment for $\mathrm{CoS}_{2}(001)$. The surface weighted $\mathrm{Co} 3 \mathrm{~d} \mathrm{e}_{\mathrm{g}}$ band exhibits only a small dispersion with wavevector, while the surface weighted Co $3 \mathrm{~d}\left(\mathrm{t}_{2 \mathrm{~g}}\right)$ bands are seen to have greater dispersion in the surface Brillouin zone along the $\langle 100\rangle$ 
direction. The calculated (001) surface band structure is seen to be consistent with experiment. Alloying with the narrow band gap semiconductor $\mathrm{FeS}_{2}$ to generate the highly spinpolarized ferromagnet $\mathrm{Fe}_{1-x} \mathrm{Co}_{x} \mathrm{~S}_{2}$ may well leave a band gap at the Fermi level in the minority spin density of states for some surfaces and surface terminations in ground state calculations. This does not imply, however, that such a surface or interface will be half-metallic at finite temperature [43-45], and it is necessary to explore first whether such high spin polarization surfaces are thermodynamically stable. The only other similar study where there has been an effort to compare an angle-resolved surface band structure, again spin integrated, to create a surface band mapping in support of a spinpolarized theoretical band structure for a very high polarization material is the example of NiMnSb(100) [46]. Spin-polarized photoemission studies are clearly indicated and needed.

\section{Acknowledgments}

This research was supported through the ONR Grant No. N00014-06-1-0616, the Nebraska Research Initiative and the NSF 'QSPINS' MRSEC (DMR-0820521) at UNL. The work at UMN was supported by the NSF MRSEC under DMR-0212302 and DMR-0819885. The Center for Advanced Microstructures and Devices is supported by the Louisiana Board of Regents.

\section{References}

[1] Zhao G L, Callaway J and Hayashibara M 1993 Phys. Rev. B 4815781

[2] Kwon S K, Youn S J and Min B I 2000 Phys. Rev. B 62357

[3] Shishidou T, Freeman A J and Asahi R 2001 Phys. Rev. B 64180401

[4] Mazin I I 2000 Appl. Phys. Lett. 773000

[5] Umemoto K, Wentzcovitch R M, Wang L and Leighton C 2006 Phys. Status Solidi b 243 2117-21

[6] Ramesha K, Seshadri R, Ederer C, He T and Subramanian M A 2004 Phys. Rev. B 70214409

[7] Wu N, Losovyj Y B, Wisbey D, Belashchenko K, Manno M, Wang L, Leighton C and Dowben P A 2007 J. Phys.: Condens. Matter 19156224

[8] Ohsawa A, Yamaguchi Y, Watanabe H and Itoh H 1976 J. Phys. Soc. Japan 40986

[9] Adachi K, Sato K and Takeda M 1969 J. Phys. Soc. Japan 26 631

[10] Wang L, Chen T Y and Leighton C 2004 Phys. Rev. B 69094412

[11] Wang L, Umemoto K, Wentzcovitch R M, Chen T Y, Chien C L, Checkelsky J G, Eckert J C, Dahlberg E D and Leighton C 2005 Phys. Rev. Lett. 94056602

[12] Wang L, Chen T Y, Chien C L and Leighton C 2006 Appl. Phys. Rev. 88232509

[13] Soulen R J, Byers J M, Osofsky M S, Nadgorny B, Ambrose T, Cheng S F, Broussard P R, Tanaka C T, Nowak J, Moodera J S, Barry A and Coey J M D 1998 Science 28285

[14] Bona G L, Meier F, Taborelli M, Bucher E and Schmidt P H 1985 Solid State Commun. $\mathbf{5 6} 391$
[15] Wolfgang G 1991 Spin- und winkelauflösende Inverse Photoemission an NiMnSb Doctoral Thesis Fachbereich Physik, Free University of Berlin

[16] Zhu W, Sincovic B, Vescovo E, Tanaka C and Moodera J S 2001 Phys. Rev. B 64060403

[17] Tanaka C T, Nowak J and Moodera J S 1999 J. Appl. Phys. 866239

[18] Caballera J A et al 1999 J. Magn. Magn. Mater. 198/199 55

[19] Borca C N, Komesu T, Jeong H, Dowben P A, Ristoiu D, Hordequin Ch, Nozières J P, Pierre J, Stadler S and Idzerda Y U 2001 Phys. Rev. B 64052409

[20] Ristoiu D, Nozières J P, Borca C N, Komesu T, Jeong H-K and Dowben P A 2000 Europhys. Lett. 49624

[21] Velev J P, Dowben P A, Tsymbal E Y, Jenkins S J and Caruso A N 2008 Surf. Sci. Rep. 63400

[22] Mazin I I 1999 Phys. Rev. Lett. 831427

[23] Dowben P A 2007 J. Phys.: Condens. Matter 19310301

[24] Nadgorny B, Mazin I I, Osofsky M, Soulen R J, Broussard P, Stroud R M, Singh D J, Harris V G, Arsenov A and Mukovskii Ya 2001 Phys. Rev. B 63184433

[25] Nadgorny B 2007 J. Phys.: Condens. Matter 19315209

[26] Fang C M, de Wijs G A and de Groot R A 2002 J. Appl. Phys. 918340

[27] Galanakis I 2002 J. Phys.: Condens. Matter 146329

[28] Galanakis I 2002 Phys. Rev. B 66012406

[29] Jenkins S J and King D A 2001 Surf. Sci. Lett. 494 L793

[30] Mollet S and Jenkins S J 2007 J. Phys.: Condens. Matter 19315214

[31] Fonin M, Pentcheva R, Dedkov Yu S, Sperlich M, Vyalikn D V, Scheffler M, Rudiger U and Guntherodt G 2005 Phys. Rev. B 72104436

[32] de Wijs G A and de Groot R A 2001 Phys. Rev. B 64020402

[33] Mavropoulos P and Galanakis I 2007 J. Phys.: Condens. Matter 19315221

[34] Picozzi S, Continenza A and Freeman A J 2003 J. Appl. Phys. 944723

[35] Jenkins S J and King D A 2002 Surf. Sci. Lett. 501 L185

[36] Picozzi S, Continenza A and Freeman A J 2003 J. Phys. Chem. Solids 641697

[37] Galanakis I, Ozdogan K and Sasioglu E 2008 J. Appl. Phys. 104083916

[38] Yu Z X, van Hove M A, Tong S Y, Wisbey D, Losovyj Y B, Wu N, Manno M, Wang L, Leighton C, Mei W N and Dowben P A 2007 J. Phys.: Condens. Matter 19156223

[39] Wu N, Sabirianov R F, Duan C-G, Mei W N, Wisbey D, Losovyj Ya B, Manno M, Leighton C, Cai E, Zhang J and Dowben P A 2008 J. Phys.: Condens. Matter 20215131

[40] Losovyj Y, Ketsman I, Morikawa E, Wang Z, Tang J and Dowben P 2007 Nucl. Instrum. Methods Phys. Res. A $\mathbf{5 8 2} 264$

[41] Takahashi T, Naitoh Y, Sato T, Kamiyama T, Yamada K, Hiraka H, Endoh Y, Usuda M and Hamada N 2001 Phys. Rev. B 63094415

[42] Losovyj Y, Morris K, Rosa L, Scott J D and Dowben P 2007 Nucl. Instrum. Methods Phys. Res. A $\mathbf{5 8 2} 258$

[43] Skomski R and Dowben P A 2002 Europhys. Lett. 58544

[44] Dowben P A and Skomski R 2004 J. Appl. Phys. 957453

[45] Dowben P A and Jenkins S J 2005 The limits to spin-polarization in finite-temperature half-metallic ferromagnets Frontiers in Magnetic Materials ed A Narlikar (Berlin: Springer) pp 295-325

[46] Correa J S, Eibl C, Rangelov G, Braun J and Donath M 2006 Phys. Rev. B 73125316 